\title{
Self-controlled Case Series Study (SCCSS): A Novel Research Method $\underline{\text { El-Gilany } \mathrm{AH}^{1^{*}}}$
}

${ }^{1}$ Professor of Public Health \& Preventive Medicine, Faculty of Medicine, Mansoura University, Mansoura 35516, Egypt

Corresponding Author: Prof. Abdel-Hady El-Gilany

Address: Professor of Public Health \& Preventive Medicine, Faculty of Medicine, Mansoura University, Mansoura 35516, Egypt.

Received date: 20 May 2019; Accepted date: 02 June 2019; Published date: o8 June 2019

Citation: El-Gilany AH, "Self-controlled case series study (SCCSS): a novel research method". Asp Biomed Clin Case Rep, vol.2, no.1: 29-32, 2019.

Copyright (C) 2019 El-Gilany AH, This is an open access article distributed under the Creative Commons Attribution License, which permits unrestricted use, distribution, and reproduction in any medium, provided the original work is properly cited.

\section{Abstract}

Self-controlled case series study (SCCSS) is a novel strategy to control between-person confounders by comparing the risk and reference periods in each patient. SCCSS investigates the association between transient exposure and an adverse event. It was developed to study adverse reactions to vaccines. Risk periods are defined during or after the exposure. Then the method finds a relative incidence i.e. the incidence in risk periods relative to the incidence in control periods. This editorial describes the definition, origins, design, assumptions, advantages, and limitations of this research method.

\section{Keywords}

Self-Controlled Case Series (SCCS); Consecutive Controlled Case Series (CCCS)

\section{Abbreviation}

Consecutive Controlled Case Series Study (CCCSS); Self-Controlled Case Series Method (SCCSM) and sometimes called case series study for simplicity.

\section{Definition}

The SCCSS is an epidemiological study design for which individuals act as their own control i.e. comparisons are made within individuals. The temporal association between transient exposure and an event is estimated. SCCSS was originally developed for evaluation of vaccine safety but has since been applied in a range of settings where exact information on the size of the population at risk is lacking or identification of an appropriate comparison group is difficult e.g. for studies of adverse effects of drugs. SCCS is best suited to acute recurrent or nonrecurrent events and transient exposures for which precise timings are available [1].

\section{Background}

Since the early 1990s, several designs have been proposed, where the comparison is not between drug users and nonusers but between time spent as a drug user and time spent as a nonuser, within the same subjects. The comparison is between different experiences in the same subjects cancel out all the potential confounders that are stable over time. This even extends to confounders that cannot be measured or are unknown (e.g. genetic factors). These designs pose the question 'why now?' instead of 'why me?' 
that is posed in a design based on other control subjects in traditional study designs [2]. SCCSM was first introduced in 1995; it was used to study the association between vaccines and acute potential adverse events $[3,4,5]$. Subsequently, the method was applied in other settings such as investigating nonacute events as autism [6] and has been used more widely in pharmaco-epidemiology (e.g. antidepressant use and the risk of hip fracture; adverse events of drugs) [7] and other areas of epidemiology.

SCCSS is a modified cohort method for estimating the relative incidence of specified events in a defined period after a transient exposure. The method is based on a retrospective cohort model applied to a defined observation period, conditionally on the number of events experienced by each individual over the observation period. The time within the exposure period is classified as at risk or as control time, in relation to exposures that are regarded as fixed [8]. The method is particularly attractive for use with data from large databases that may have been assembled for reasons unrelated to the study, as is the case with administrative databases. In such databases, information on important confounders, such as whether a person smokes or not, or their socioeconomic background, or general state of health, may not be available.

SCCSS differs from the consecutive case series because of the latter reports on multiple cases without control. In contrast, an SCCSS includes cases diagnosed during the exposure period and compared to cases diagnosed during the control period in each individual participant. Thus, the SCCSS design has better internal validity than a consecutive case series $[7,8]$.

\section{SCCSS Design}

The self-controlled case series (SCCS) can best be understood as cohort logic applied to a case-only design. SCCS is a cohort study (retrospective, prospective or bidirectional) in which individuals experience successive 'at risk' periods and 'not at risk' as control periods over a defined follow-up period $[3,9]$.
Other important features of standard selfcontrolled case series are that the exposure history occurring after the case-defining event is included in the estimates and (by consequence) that more than one occurrence of the end-point is allowed. By including follow-up after the outcome, the selfcontrolled case series can be viewed as a bidirectional design. The analysis is confined to subjects who become cases $[10,11]$.

\section{Steps}

$[3,8,9]$

1. Define cases and exposure. Data will be collected on cases (who have the event) only.

2. Define the observation periods: the study period for each individual is at risk of exposure (full history on the timing of events and exposure are available). Select this period so as to maximize the chance of exposure (e.g. study of idiopathic thrombocytopenic purpura and MMR, the study period is the second years which corresponds to the age range of primary MMR vaccination).

3. The exposure risk periods should be defined a priori (e.g. vaccination dates of each case), during which individuals are hypothesized to be at increased (or reduced) risk of the event of interest after (or before) exposure. All remaining time within the observation period constitutes baseline exposure periods, to which the exposure risk periods will be compared. Multiple exposure risk periods can be included as it may be necessary to capture either multiple doses or varying exposure-related risk using several risk periods. Cases are all or a random sample of individuals with at least one event (independent recurrences).

4. "Map" events in relation to the different periods identified.

5. Estimate the relative incidence of events in prespecified exposure risk periods compared with baseline periods, while taking into account the effect of any time-varying confounders such as age groups. Comparisons are not made between individuals as in a cohort or case-control study; estimation is within individuals. 


\section{SCCSS Assumptions}

The key assumptions of the SCCS method are that (1) events are recurrent and independent, or are unique and rare over the observation periods; (2) the occurrence of an event must not alter the probability of subsequent exposure. This requirement is violated when events increase the mortality rate, since censoring of the observation periods is then event dependent; (3) the occurrence of the event of interest must not censor or affect the observation period; (4) event rates are constant within intervals; and (5) the observation periods should be independent of event times $[1,3,12,13,14,15]$.

\section{Advantages}

$[8,11,13,14,16,17]$

1. It is based only on cases and provides consistent estimates of the relative incidence.

2. Effective control for all fixed confounders that are stable over time (self-controlled). This even extends to confounders that are unmeasured or unknown. This is particularly useful for studies conducted in administrative databases, in which relevant covariate information may be limited.

3. Overcome some of the difficulties and pitfalls of control selection.

4. The number of subjects required for a selfcontrolled study tends to be smaller than for a conventional approach. Only cases are required, which reduces efforts, time and cost of data collection. It can produce results that are statistically and clinically valid with far fewer patients

5. Temporal variation in the event rate is modeled as in a cohort study

6. Power is often good

7. May be used with indefinite drug use

8. Adjusts for trends in exposure

9. In surveillance of vaccine adverse events traditional cohort and case-control designs may not be feasible for studying vaccines with coverage approaching $100 \%$ as it would be difficult to recruit unvaccinated controls, and safety surveillance systems typically only collect data for individuals who reported an adverse event thought to be related to vaccination.

10. Minimal ethical considerations

\section{Limitations of SCCSS}

$[8,11,13,17,18,19]$.

1. It requires that the probability of exposure is not affected by the occurrence of an outcome event.

2. For non-recurrent events, the method works only when the event risk is small over the observation period.

3. It does not produce estimates of absolute incidence, only estimates of relative incidence.

4. It requires variability in the time or age of the event: if all events were to happen at exactly the same age, then the method would fail.

5. The occurrence of an event should not appreciably increase mortality (recently a complex methodology was used to overcome this condition)

6. The occurrence of an event should not affect subsequent exposure history (recently a complex methodology was used to overcome this condition)

7. The major limitation of the self-controlled designs is that the effect of exposure has to be transient. For research questions in which the key is cumulative rather than current exposure, conventional case-control or cohort approaches are better suited. SCCS is more vulnerable to misclassification bias than the conventional case-control approach.

8. The use of subjects as their own controls already adjusts for fixed factors, but it does not account for the variations over time. The adjustment for time trends in the case-time-control studies is limited to the measured variables.

9. Liable to selection and information bias, as in any case-control study.

10. Referral bias in clinic-based studies and the sample may not be representative of the broader population.

11. The use of the subjects as their own controls has been limited in clinical trials, for many reasons. One of them is the ethical worry of allowing patients with placebo, only, during the reference period (non-exposure period).

12. It assumes that treatment effects are homogeneous across subjects. However, there may be variations in how the treatment is applied across participants. This is particularly 
Citation: El-Gilany AH, "Self-controlled case series study (SCCSS): a novel research method". Asp Biomed Clin Case Rep, vol.2, no.1: 29-32, 2019 .

important in behavioral interventions which are individualized, and continuously evaluated, and altered based on the individual's response.

13. Complicated data processing and analysis

\section{References}

[1] Petersen I, Douglas I, Whitaker H, "Self-controlled case series methods: an alternative to standard epidemiological study designs”. BMJ, vol.354: i4515, 2016.

[2] Maclure M, “'Why me?' versus 'why now?'differences between operational hypotheses in case-control versus case-crossover studies". Pharmacoepidemiol Drug Saf, vol.16, no.8: 850-53, 2007.

[3] Farrington CP, "Relative incidence estimation from case series for vaccine safety evaluation”. Biometrics, vol.51, no.1: 228-35, 1995.

[4] Farrington CP, Pugh S, Colville A, et al., "A new method for active surveillance of adverse events from diphtheria tetanus pertussis and measles mumps rubella vaccines". Lancet, vol.345, no.8949: 567-69, 1995 .

[5] Farrington CP, Nash J and Miller E, "Case series analysis of adverse reactions to vaccines: a comparative evaluation”. Am J Epidemiol, vol.143, no.11: 1165-73, 1996.

[6] Taylor B, Miller E, Farrington CP, et al., "Autism and measles, mumps and rubella vaccine: no epidemiological evidence for a causal association". Lancet, vol.353: 2026-29, 1999.

[7] Hubbard R, Farrington P, Smith C, et al., "Exposure to Tricyclic and selective serotonin reuptake inhibitor antidepressants and the risk of hip fracture”. Am J Epidemiol, vol.158, no.1: 77-84, 2003.

[8] Musonda P, Farrington CP, Whitaker HJ, "Sample sizes for self-controlled case series studies”. Stat Med, vol.25, no.15: 2618-31, 2006.

[9] Weldeselassie YG, Whitaker HJ, Farrington CP, "Use of the self-controlled case-series method in vaccine safety studies: review and recommendations for best practice". Epidemiol Infect, vol.139, no.12: 1805-17, 2011.

[10] Maclure M, Fireman B, Nelson JC, et al., "When should case-only designs be used for safety monitoring of medical products?”. Pharmacoepidemiol Drug Saf, vol.21, suppl.1: 50-61, 2012.

[11] Hallas J., Pottegard A, "Use of self-controlled designs in pharmacoepidemiology". J Intern Med, vol.275, no.6: 581-89, 2014.

[12] Farrington CP, Whitaker HJ, "Semiparametric analysis of case series data". Journal of the Royal Statistical Society: Series C, vol.55, no.5: 553-94, 2006.

[13] Whitaker HJ, Farrington CP, Spiessens B, et al., "Tutorial in biostatistics: The self-controlled case series method". Stat Med, vol.25, no.10: 1768-97, 2006.

[14] Whitaker HJ, Hocine MN, Farrington CP, "The methodology of self-controlled case series studies". Stat Methods Med Res, vol.18, no.1: 7-26, 2009.

[15] Farrington CP, Anaya-Izquierdo K, Whitaker HJ, et al., "Self-controlled case series analysis with eventdependent observation periods". Journal of the American Statistical Association, vol.106, no.494: 41726, 2011.

[16] Hawken S, Potter BK, Little J, et al., "The use of relative incidence ratios in self-controlled case series studies: an overview”. BMC Med Res Methodol, vol.16, no.1: 126, 2016.

[17] Farrington P, Whitaker H, Weldeselassie YG, "Self-Controlled Case Series Studies. A Modelling Guide with R”: 1-362, 2018.

[18] Greenland S, "Confounding and exposure trends in case crossover and case-time-control designs". Epidemiology, vol.7, no.3: 231-39, 1996.

[19] Andrews N, "Population-based post-licensure safety surveillance”. Public Health England: 2014. 